\title{
Spondylarthritiden - IL-17A-Inhibition auch bei relevanten Komorbiditäten effektiv
}

Eine breite Datenbasis aus randomisierten Studien belegt die gute Wirksamkeit des direkten Interleukin 17A (IL-17A)-Inhibitors Secukinumab auf alle relevanten Endpunkte. Das Sicherheitsprofil ist günstig.

Quelle: Dr. Wiebke Kathmann, Symposium Novartis Pharma „Innovative anti-IL-17A-Therapie bei Spondylarthritiden: Wo stehen wir, wo wollen wir hin?" anlässlich des DGRh-Kongresses 2018, Mannheim, 21.09.2018 der Fälle betroffen (Kiltz U et al. EULAR 2018, Abstract SAT0299). Der PsoriasisSchweregrad (PASI) verbesserte sich unter Secukinumab von 5,0 zu Beginn auf 0,0 nach 52 Wochen. Bei der Hälfte der Patienten war die Haut abgeheilt.

) Extraartikuläre Manifestationen wie Uveitis oder Psoriasis erhöhen die Krankheitslast der Patienten

Umgekehrt war eine Uveitis erwartungsgemäß bei AS-Patienten häufiger (6,2 versus $0,9 \%)$. Unter der Therapie entwickelten nur ein AS- und zwei PsA-Patienten erstmalig eine Uveitis. Eine kardiovaskuläre Erkrankung war waren bei PsA-Patienten häufiger (KHK: 8,9 versus 3,5\%, Herzinsuffizienz: 3,3 versus $0,7 \%$, Schlaganfall 2,4 versus $0,0 \%)$. Unter der 52 -wöchigen Therapie mit dem IL-17A-Inhibitor ver- schlechterten sich die kardiovaskulären Erkrankungen nicht. Nur bei zwei PsA-Patienten trat im Verlauf eine KHK bzw. Herzinsuffizienz auf.

Depressionen waren zu Beginn in beiden Gruppen häufig (PsA: 15,4\%, AS: 12,2\%). Am Ende der Therapiephase hatten sie sich deutlich gebessert (Beck-Depressions-Inventar II von 12,0 auf 6,0 bzw. 9,0 auf 6,0 ). Somit konnten unter der Secukinumab-Therapie die kardiovaskulären Komorbiditäten stabil gehalten werden. Die für Patienten relevanten Symptome Psoriasis und Depression verbesserten sich.

rheuma plus $2018 \cdot 17: 196$ https://doi.org/10.1007/s12688-0180222-y

(c) Springer-Verlag GmbH Austria, ein Teil von Springer Nature 2018

Hier steht eine Anzeige. 